\title{
Novel Pyrazole-Hydrazone Derivatives Containing an Isoxazole Moiety: Design, Synthesis, and Antiviral Activity
}

\author{
Zaibo Yang ${ }^{1, *}$, Pei $\mathrm{Li}^{2}$ and Xiuhai Gan ${ }^{3}$ \\ 1 School of Chemistry and Chemical Engineering, Qiannan Normal University for Nationalities, \\ Guizhou, Duyun 558000, China \\ 2 Qiandongnan Engineering and Technology Research Center for Comprehensive Utilization of National \\ Medicine, Kaili University, Guizhou, Kaili 556011, China; pl19890627@126.com \\ 3 College of Chemistry and Life Science, Guizhou Normal College, Guizhou, Guiyang 550018, China; \\ gxh200719@163.com \\ * Correspondence: qnsyyzb27001@sgmtu.edu.cn; Tel.: +86-85-4873-7046
}

Received: 19 June 2018; Accepted: 18 July 2018; Published: 20 July 2018

\begin{abstract}
In this study, a series of novel pyrazole-hydrazone derivatives containing an isoxazole moiety were synthesized. Antiviral bioassays indicated that some of the title compounds exhibited better in vivo antiviral activities against tobacco mosaic virus (TMV). In particular, compounds 6a, 6c and $\mathbf{6 q}$ exhibited the best curative activity, protection activity, and inactivation activity against TMV, respectively, which were superior to those of Ningnanmycin. This study demonstrated that this series of novel pyrazole-hydrazone derivatives containing an isoxazole amide moiety could effectively control TMV.
\end{abstract}

Keywords: pyrazole-hydrazone; isoxazole; antiviral activity; tobacco mosaic virus

\section{Introduction}

Plant viruses cause diseases in a wide range of crop plant species and annually contribute to an estimated $\$ 600$ billion in economic loss worldwide [1]. In recent years, progress has been made in screening for high-activity antiviral agents in natural products in China. For example, Ningnanmycin, isolated from Strepcomcesnoursei var. Xichangensis, was found to be more effective than existing products in the treatment of plant viruses such as tobacco mosaic virus (TMV) and cucumber mosaic virus (CMV) [2]. Meanwhile, Ribavirin, a successful antiviral agent, is widely used to prevent TMV disease. However, the use of Ningnanmycin and Ribavirin in field trials is unsuitable because of its unsatisfactory curative rates and high control costs [3]. Therefore, identification of new effective antiviral agents remains a significant challenge [4].

In recent years, literature revealed that pyrazole derivatives had a wide range of biological activities, such as antibacterial [5-7], fungicidal [8-10], insecticidal [10-13], antiviral [14,15], and herbicidal [16,17] activities. In our previous work, we reported a series of pyrazole derivatives which had better insecticidal activity against Plutella xylostella [18]. Meanwhile, hydrazone, a highly efficient pharmacophore, has attracted more and more attention in the pesticides areas due to its broad-activities, such as antibacterial [19], fungicidal [20,21], insecticidal [20,22,23], and antiviral [20,24] activities. A few of the pyrazole-hydrazone derivatives have also been reported and possess various biological activities such as antibacterial [25], antifungal [21], anticancer [26], antioxidant [27], anti-inflammatory [28], antitumor and antiangiogenesis [29] and antiplatelet activities [30]. In addition, isoxazole, a five membered heterocyclic ring, was an important scaffold for synthesis of various 
natural compounds and their congeners and had a broad spectrum of pharmacological activities like antibacterial [31], antifungal [9,32,33], insecticide [34,35], and antiviral [36] activities.

Motivated by the above-mentioned findings and in continuation of our investigation, to discover new potentially active agents, in this study, a series of novel pyrazole-hydrazone derivatives containing an isoxazole moiety were designed and synthesized using a 3-substituted phenyl-5-methyl isoxazole-4-carboxylic acid as the starting material. The antiviral bioassay results indicated that most of the title compounds exhibited excellent in vivo antiviral activities against TMV. To the best of our knowledge, it is the first report on pyrazole-hydrazone derivatives containing an isoxazole moiety with potent antiviral activity against TMV.

\section{Results and Discussion}

\subsection{Chemistry}

Using 3-(2-chlorophenyl)-5-methyl isoxazole-4-carboxylic acid and 3-(2,6-dichlorophenyl)-5methyl isoxazole-4-carboxylic acid as the starting materials, as shown in Scheme 1, the target compounds 6a-6r were obtained in five steps with yields of $85.9-91.2 \%$. The physical characteristics, IR, ${ }^{1} \mathrm{H}-\mathrm{NMR},{ }^{13} \mathrm{C}-\mathrm{NMR}$, and elemental analysis data for all the synthesized compounds are shown below. The ${ }^{1} \mathrm{H}-\mathrm{NMR}$ and ${ }^{13} \mathrm{C}-\mathrm{NMR}$ for all the synthesized compounds are shown in Supplementary Materials.
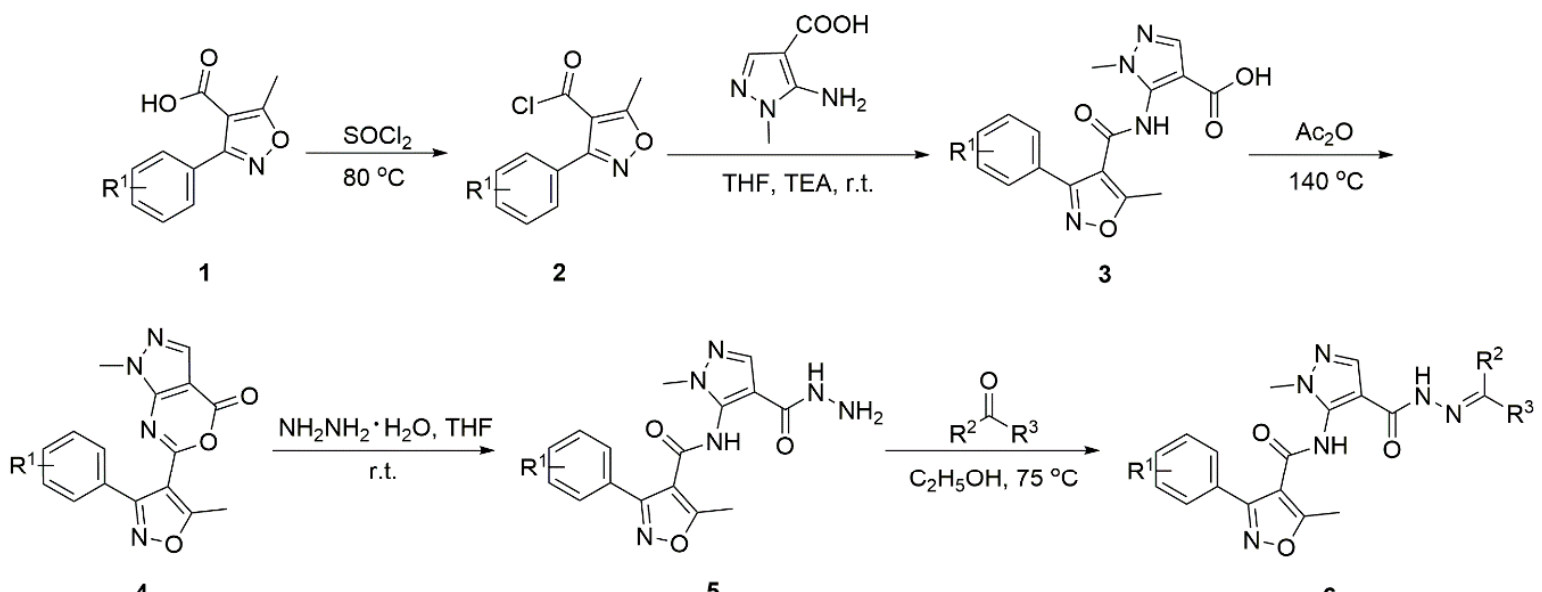
4

\begin{abstract}
6a: $\mathrm{R}^{1}=2-\mathrm{Cl}, \mathrm{R}^{2}=\mathrm{H}, \mathrm{R}^{3}=4-\mathrm{Cl}-\mathrm{Ph}$ 6b: $\mathrm{R}^{1}=2-\mathrm{Cl}, \mathrm{R}^{2}=\mathrm{H}, \mathrm{R}^{3}=4-\mathrm{Br}-\mathrm{Ph}$ 6c: $\mathrm{R}^{1}=2-\mathrm{Cl}, \mathrm{R}^{2}=\mathrm{H}, \mathrm{R}^{3}=2-\mathrm{Br}-\mathrm{Ph}$ 6d: $\mathrm{R}^{1}=2-\mathrm{Cl}, \mathrm{R}^{2}=\mathrm{H}, \mathrm{R}^{3}=2-\mathrm{Cl}-\mathrm{Ph}$ 6e: $\mathrm{R}^{1}=2-\mathrm{Cl}, \mathrm{R}^{2}=\mathrm{H}, \mathrm{R}^{3}=4-\mathrm{Me}-\mathrm{Ph}$ 6f: $\mathrm{R}^{1}=2-\mathrm{Cl}, \mathrm{R}^{2}=\mathrm{H}, \mathrm{R}^{3}=2-\mathrm{F}-\mathrm{Ph}$ 6g: $\mathrm{R}^{1}=2-\mathrm{Cl}, \mathrm{R}^{2}=\mathrm{H}, \mathrm{R}^{3}=$ Thienyl 6h: $R^{1}=2-C l, R^{2}=H, R^{3}=2,4-d i C l-P h$ 6i: $\mathrm{R}^{1}=2-\mathrm{Cl}, \mathrm{R}^{2}=\mathrm{H}, \mathrm{R}^{3}=4-\mathrm{NO}_{2}-\mathrm{Ph}$
\end{abstract}

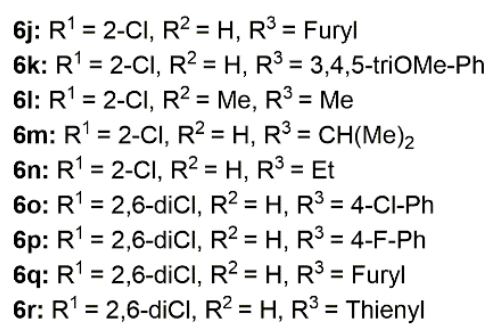

Scheme 1. Synthetic route of the title compounds $6 \mathbf{a}-6 \mathbf{r}$.

Data for N-(4-(2-(4-chlorobenzylidene)hydrazinecarbonyl)-1-methyl-1H-pyrazol-5-yl)-3-(2-chlorophenyl)-5methylisoxazole-4-carboxamide (6a). White solid; m.p. $141-143{ }^{\circ} \mathrm{C}$; yield $87.9 \%$; ${ }^{1} \mathrm{H}-\mathrm{NMR}(500 \mathrm{MHz}$, Dimethylsulfoxide- $d_{6}$ (DMSO- $\left.\left.d_{6}\right), \mathrm{ppm}\right) \delta: 11.54(\mathrm{~s}, 1 \mathrm{H}$, isoxazole-CONH-), $10.32(\mathrm{~s}, 1 \mathrm{H}$, pyrazole-CONH-), $8.31(\mathrm{~s}, 1 \mathrm{H},-\mathrm{N}=\mathrm{CH}-), 8.01(\mathrm{~s}, 1 \mathrm{H}$, pyrazole-H), 7.74-7.47 (m, 8H, Ar-H), $3.65(\mathrm{~s}$, $3 \mathrm{H}$, pyrazole- $\left.\mathrm{CH}_{3}\right), 2.85\left(\mathrm{~s}, 3 \mathrm{H},-\mathrm{CH}_{3}\right) .{ }^{13} \mathrm{C}-\mathrm{NMR}\left(125 \mathrm{MHz}\right.$, DMSO- $\left.d_{6}, \mathrm{ppm}\right) \delta: 171.53,160.66,158.43$, $147.02,145.78,144.08,140.42,140.78,139.24,137.88,137.67,132.84,132.15,130.25,130.13,129.97,127.94$, 127.50, 127.26, 113.72, 109.57, 36.66, 12.99. IR $\left(\mathrm{KBr}, \mathrm{cm}^{-1}\right) v: 3404.36,3282.84,2933.73,1716.65,1699.29$, 1683.86, 1653.00, 1635.64, 1627.92, 1595.13, 1575.84, 1558.48, 1541.12, 1521.84, 1506.41, 1489.08, 1473.62, 
$1458.18,1436.97,1411.89,1375.25,1303.88,1253.75,1219.01$. Anal. Calc. for $\mathrm{C}_{23} \mathrm{H}_{18} \mathrm{Cl}_{2} \mathrm{~N}_{6} \mathrm{O}_{3}: \mathrm{C}, 55.55 \%$; H, 3.65\%; N, 16.90\%; Found: C, 55.81\%; H, 3.79\%; N, $17.08 \%$.

Data for 3-(2-chlorophenyl)-N-(4-(2-(4-bromobenzylidene)hydrazinecarbonyl)-1-methyl-1H-pyrazol-5-yl)-5methylisoxazole-4-carboxamide (6b). White solid; m.p. 236-237 ${ }^{\circ} \mathrm{C}$; yield $90.1 \%$; ${ }^{1} \mathrm{H}-\mathrm{NMR}(500 \mathrm{MHz}$, DMSO- $\left.d_{6}, \mathrm{ppm}\right) \delta: 11.57(\mathrm{~s}, 1 \mathrm{H}$, isoxazole-CONH-), $10.35(\mathrm{~s}, 1 \mathrm{H}$, pyrazole-CONH-), $8.29(\mathrm{~s}, 1 \mathrm{H}$, $-\mathrm{N}=\mathrm{CH}-), 8.01(\mathrm{~s}, 1 \mathrm{H}$, pyrazole-H), 7.85-7.85 (d, 1H, J = 3.45 Hz, Ar-H), 7.66-7.45 (m, 7H, Ar-H), 3.66 $\left(\mathrm{s}, 3 \mathrm{H}\right.$, pyrazole- $\left.\mathrm{CH}_{3}\right), 2.86\left(\mathrm{~s}, 3 \mathrm{H},-\mathrm{CH}_{3}\right) .{ }^{13} \mathrm{C}-\mathrm{NMR}\left(125 \mathrm{MHz}, \mathrm{DMSO}-d_{6}, \mathrm{ppm}\right) \delta: 171.46,160.67$, $158.38,145.62$, 137.80, 134.21, 132.87, 132.38, 132.36, 132.17, 132.16, 131.80, 130.14, 129.37, 129.19, 127.96, 127.93, 123.57, 113.57, 109.52, 36.55, 12.99. IR $\left(\mathrm{KBr}^{\mathrm{cm}}{ }^{-1}\right) v: 3444.87,326.91,2933.73,1716.65,1699.29$, 1683.86, 1662.64, 1653.00, 1635.64, 1608.63, 1575.84, 1558.48, 1541.12, 1521.84, 1506.41, 1489.08, 1473.62, $1458.18,1436.97,1429.25,1398.39,1319.31,1301.95$. Anal. Calc. for $\mathrm{C}_{23} \mathrm{H}_{18} \mathrm{ClBrN}_{6} \mathrm{O}_{3}: \mathrm{C}, 50.99 \%$; $\mathrm{H}$, $3.35 \%$; N, 15.51\%; Found: C, 51.09\%; H, 3.39\%; N, 15.55\%.

Data for 3-(2-chlorophenyl)-N-(4-(2-(2-bromobenzylidene)hydrazinecarbonyl)-1-methyl-1H-pyrazol-5-yl)-5methylisoxazole-4-carboxamide (6c). White solid; m.p. $181-183{ }^{\circ} \mathrm{C}$; yield $89.2 \%$; ${ }^{1} \mathrm{H}-\mathrm{NMR}(500 \mathrm{MHz}$, DMSO- $\left.d_{6}, \mathrm{ppm}\right) \delta: 11.69(\mathrm{~s}, 1 \mathrm{H}$, isoxazole-CONH-), $10.30(\mathrm{~s}, 1 \mathrm{H}$, pyrazole-CONH-), $8.61(\mathrm{~s}, 1 \mathrm{H}$, $-\mathrm{N}=\mathrm{CH}-), 7.97(\mathrm{~s}, 1 \mathrm{H}$, pyrazole-H), $7.91(\mathrm{~d}, 1 \mathrm{H}, J=7.45 \mathrm{~Hz}, \mathrm{Ar}-\mathrm{H}), 7.63(\mathrm{~d}, 1 \mathrm{H}, J=8.00 \mathrm{~Hz}, \mathrm{Ar}-\mathrm{H})$, 7.54-7.38 (m, 5H, A-H), $7.31(\mathrm{t}, 1 \mathrm{H}, J=14.85 \mathrm{~Hz}, \mathrm{Ar}-\mathrm{H}), 3.59\left(\mathrm{~s}, 3 \mathrm{H}\right.$, pyrazole- $\left.\mathrm{CH}_{3}\right), 2.79\left(\mathrm{~s}, 3 \mathrm{H},-\mathrm{CH}_{3}\right)$. ${ }^{13} \mathrm{C}-\mathrm{NMR}\left(125 \mathrm{MHz}\right.$, DMSO- $\left.d_{6}, \mathrm{ppm}\right) \delta: 160.56,158.40,158.18,152.48,147.87,144.98,137.96,137.71$, $133.63,132.75,132.10,131.86,130.07,128.58,127.88,127.71,127.63,123.88,113.69,113.58,112.30,36.61$, 12.91. IR $\left(\mathrm{KBr}, \mathrm{cm}^{-1}\right) v: 3446.79,3298.28,2933.73,1716.65,1699.29,1683.86,1670.35,1653.00,1635.64$, 1575.84, 1558.48, 1541.12, 1521.84, 1506.41, 1489.08, 1473.62, 1458.18, 1436.97, 1411.89, 1398.39, 1361.74, 1338.60, 1319.31, 1298.09, 1253.73, 1220.94. Anal. Calc. for $\mathrm{C}_{23} \mathrm{H}_{18} \mathrm{ClBrN}_{6} \mathrm{O}_{3}: \mathrm{C}, 50.99 \% ; \mathrm{H}, 3.35 \%$; N, $15.51 \%$; Found: C, $51.06 \%$; H, 3.41\%; N, 15.57\%.

Data for 3-(2-chlorophenyl)-N-(4-(2-(2-chlorobenzylidene)hydrazinecarbonyl)-1-methyl-1H-pyrazol-5-yl)-5methylisoxazole-4-carboxamide (6d). White solid; m.p. 170-171 ${ }^{\circ} \mathrm{C}$; yield $88.7 \%$; ${ }^{1} \mathrm{H}-\mathrm{NMR}(500 \mathrm{MHz}$, DMSO- $\left.d_{6}, \mathrm{ppm}\right) \delta: 11.64(\mathrm{~s}, 1 \mathrm{H}$, isoxazole-CONH-), $10.28(\mathrm{~s}, 1 \mathrm{H}$, pyrazole-CONH-), $8.66(\mathrm{~s}, 1 \mathrm{H}$, $\mathrm{N}=\mathrm{CH}-), 7.97\left(\mathrm{~s}, 1 \mathrm{H}\right.$, pyrazole-H), 7.53-7.37 $(\mathrm{m}, 8 \mathrm{H}, \mathrm{Ar}-\mathrm{H}), 3.59\left(\mathrm{~s}, 3 \mathrm{H}\right.$, pyrazole- $\left.\mathrm{CH}_{3}\right), 2.79(\mathrm{~s}, 3 \mathrm{H}$, - $\left.\mathrm{CH}_{3}\right) .{ }^{13} \mathrm{C}-\mathrm{NMR}\left(125 \mathrm{MHz}\right.$, DMSO- $d_{6}$, ppm) $\delta: 171.52,160.65,158.51,142.75,140.37,138.09,137.70$, $133.55,132.82,132.16,131.88,131.73,130.46,130.14,128.17,127.95,127.84,127.33,127.14,113.71,109.36$, 36.66, 12.97. IR $\left(\mathrm{KBr}, \mathrm{cm}^{-1}\right)$ v: 3444.87, 3286.70, 2933.73, 1716.65, 1695.43, 1683.86, 1670.35, 1653.00, 1635.64, 1575.84, 1558.48, 1541.12, 1521.84, 1506.41, 1498.69, 1473.62, 1458.18, 1436.97, 1419.61, 1398.39, 1361.74, 1340.53, 1319.31, 1251.80, 1219.01. Anal. Calc. for $\mathrm{C}_{23} \mathrm{H}_{18} \mathrm{Cl}_{2} \mathrm{~N}_{6} \mathrm{O}_{3}: \mathrm{C}, 55.55 \% ; \mathrm{H}, 3.65 \%$; , $16.90 \%$; Found: C, $55.70 \% ; \mathrm{H}, 3.79 \%$; N, 17.24\%.

Data for 3-(2-chlorophenyl)-5-methyl-N-(1-methyl-4-(2-(4-methylbenzylidene)hydrazinecarbonyl)-1H-pyrazol -5-yl)isoxazole-4-carboxamide (6e). White solid; m.p. $221-223{ }^{\circ} \mathrm{C}$; yield $89.8 \% ;{ }^{1} \mathrm{H}-\mathrm{NMR}(500 \mathrm{MHz}$, DMSO- $\left.d_{6}, \mathrm{ppm}\right) \delta: 11.38(\mathrm{~s}, 1 \mathrm{H}$, isoxazole-CONH-), $10.27(\mathrm{~s}, 1 \mathrm{H}$, pyrazol-CONH-), $8.24(\mathrm{~s}, 1 \mathrm{H}$, -N=CH-), $7.97(\mathrm{~s}, 1 \mathrm{H}$, pyrazole-H), 7.55-7.42 (m, 6H, Ar-H), $7.22(\mathrm{~s}, 1 \mathrm{H}, \mathrm{Ar}-\mathrm{H}), 7.20(\mathrm{~s}, 1 \mathrm{H}, \mathrm{Ar}-\mathrm{H}), 3.62$ (s, 3H, pyrazole- $\left.\mathrm{CH}_{3}\right), 2.81\left(\mathrm{~s}, 3 \mathrm{H},-\mathrm{CH}_{3}\right), 2.28\left(\mathrm{~s}, 3 \mathrm{H}, \mathrm{Ar}-\mathrm{CH}_{3}\right) .{ }^{13} \mathrm{C}-\mathrm{NMR}\left(125 \mathrm{MHz}, \mathrm{DMSO}-\mathrm{d}_{6}, \mathrm{ppm}\right)$ $\delta$ : 193.16, 171.80, 171.53, 163.39, 160.66, 158.43, 147.02, 145.78, 144.08, 140.42, 140.28, 140.08, 139.24, $137.88,137.67,134.52,132.84,132.15,130.25,130.13,129.97,129.65,127.94,127.50,127.26,113.72,109.57$, 108.03, 36.66, 21.55, 12.99. IR (KBr, cm $\left.{ }^{-1}\right)$ v: 3444.87, 3203.76, 3030.17, 2933.73, 1699.29, 1683.86, 1662.64, 1653.00, 1635.64, 1602.85, 1577.77, 1558.48, 1541.12, 1521.84, 1506.41, 1473.62, 1456.26, 1436.97, 1409.96, 1398.39, 1375.25, 1301.95, 1246.02, 1219.01. Anal. Calc. for $\mathrm{C}_{24} \mathrm{H}_{21} \mathrm{ClN}_{6} \mathrm{O}_{3}: \mathrm{C}, 60.44 \% ; \mathrm{H}, 4.44 \% ; \mathrm{N}$, $17.62 \%$; Found: C, $60.53 \%$; H, $4.48 \%$; N $17.73 \%$.

Data for 3-(2-chlorophenyl)-N-(4-(2-(2-fluorobenzylidene)hydrazinecarbonyl)-1-methyl-1H-pyrazol-5-yl)-5methylisoxazole-4-carboxamide (6f). White solid; m.p. $180-182{ }^{\circ} \mathrm{C}$; yield $88.3 \%$; ${ }^{1} \mathrm{H}-\mathrm{NMR}(500 \mathrm{MHz}$, DMSO- $\left._{6}, \mathrm{ppm}\right) \delta: 11.62(\mathrm{~s}, 1 \mathrm{H}$, isoxazole-CONH-), $10.36(\mathrm{~s}, 1 \mathrm{H}$, pyrazole-CONH-), $8.57(\mathrm{~s}, 1 \mathrm{H}$, $-\mathrm{N}=\mathrm{CH}-), 8.02(\mathrm{~s}, 1 \mathrm{H}$, pyrazole-H), 7.93-7.90 (t, 1H, $J=16.60 \mathrm{~Hz}, \mathrm{Ar}-\mathrm{H}), 7.60-7.28(\mathrm{~m}, 7 \mathrm{H}, \mathrm{Ar}-\mathrm{H}), 3.66$ 
(s, 3H, pyrazole- $\left.\mathrm{CH}_{3}\right), 2.85\left(\mathrm{~s}, 3 \mathrm{H},-\mathrm{CH}_{3}\right) .{ }^{13} \mathrm{C}-\mathrm{NMR}\left(125 \mathrm{MHz}, \mathrm{DMSO}-d_{6}, \mathrm{ppm}\right) \delta: 171.25,160.15$ (d, 1C, 2-F-Ph-C), 158.14, 139.27, 137.76, 137.41, 132.54, 132.04, 131.88, 129.86, 127.65, 127.54, 126.77, $126.49,125.21,122.11,116.33,116.18,113.43,109.10,36.42,12.69$. IR $\left(\mathrm{KBr}_{\mathrm{cm}}{ }^{-1}\right) v: 3444.87,3167.12$, 2933.73, 1699.29, 1683.86, 1653.00, 1635.64, 1616.35, 1602.85, 1575.84, 1558.48, 1541.12, 1521.84, 1506.41, 1489.08, 1473.62, 1436.97, 1419.61, 1398.39, 1379.10, 1363.67, 1319.31, 1242.16, 1215.15. Anal. Calc. for $\mathrm{C}_{23} \mathrm{H}_{18} \mathrm{ClFN}_{6} \mathrm{O}_{3}$ : C, $57.45 \%$; H, 3.77\%; N, $17.48 \%$; Found: $\mathrm{C}, 57.51 \% ; \mathrm{H}, 3.96 \% ; \mathrm{N}, 17.53 \%$.

Data for 3-(2-chlorophenyl)-5-methyl-N-(1-methyl-4-(2-(thiophen-2-ylmethylene)hydrazine carbonyl)-1H -pyrazol-5-yl)isoxazole-4 -carboxamide (6g). White solid; m.p. $225-227{ }^{\circ} \mathrm{C}$; yield $89.6 \%$; ${ }^{1} \mathrm{H}-\mathrm{NMR}(500 \mathrm{MHz}$, DMSO- $\left.d_{6}, \mathrm{ppm}\right) \delta: 11.44(\mathrm{~s}, 1 \mathrm{H}$, isoxazole-CONH-), $10.32(\mathrm{~s}, 1 \mathrm{H}$, pyrazole-CONH-), $8.54(\mathrm{~s}, 1 \mathrm{H}$, $-\mathrm{N}=\mathrm{CH}-), 8.21(\mathrm{~s}, 1 \mathrm{H}$, pyrazole-H), $7.98(\mathrm{~s}, 1 \mathrm{H}, \mathrm{Ar}-\mathrm{H}), 7.65-7.14(\mathrm{~m}, 7 \mathrm{H}, \mathrm{Ar}-\mathrm{H}$, thiophen-H), 3.65 $\left(\mathrm{s}, 3 \mathrm{H}\right.$, pyrazole- $\left.\mathrm{CH}_{3}\right), 2.85\left(\mathrm{~s}, 3 \mathrm{H},-\mathrm{CH}_{3}\right) .{ }^{13} \mathrm{C}-\mathrm{NMR}\left(125 \mathrm{MHz}, \mathrm{DMSO}-d_{6}, \mathrm{ppm}\right) \delta: 171.34,160.60$, 158.32, 142.16, 139.72, 137.72, 137.62, 132.80, 132.16, 131.25, 130.75, 130.14, 129.26, 128.85, 128.57, 128.38, $127.96,113.68,109.11,36.64,12.99$. IR $\left(\mathrm{KBr}_{\mathrm{cm}}{ }^{-1}\right) v: 3446.79,3263.56,2929.87,1699.29,1683.86,1653.00$, 1635.64, 1595.13, 1577.77, 1558.48, 1541.12, 1521.84, 1506.41, 1498.69, 1473.62, 1436.97, 1419.61, 1398.39, 1375.25, 1317.38, 1300.02, 1251.80, 1224.80. Anal. Calc. for $\mathrm{C}_{21} \mathrm{H}_{17} \mathrm{ClN}_{6} \mathrm{O}_{3} \mathrm{~S}: \mathrm{C}, 53.79 \% ; \mathrm{H}, 3.65 \%$; , $17.92 \%$; Found: C, $54.08 \%$; H, 4.02\%; N, $17.96 \%$.

Data for 3-(2-chlorophenyl)-N-(4-(2-(2,4-dichlorobenzylidene)hydrazinecarbonyl)-1-methyl-1H-pyrazol-5-yl) -5-methylisoxazole-4-carboxamide (6h). White solid; m.p. $189-190{ }^{\circ} \mathrm{C}$; yield $87.9 \%$; ${ }^{1} \mathrm{H}-\mathrm{NMR}(500 \mathrm{MHz}$, DMSO- $\left.d_{6}, \mathrm{ppm}\right) \delta: 11.77(\mathrm{~s}, 1 \mathrm{H}$, isoxazole-CONH-), $10.39(\mathrm{~s}, 1 \mathrm{H}$, pyrazole-CONH-), $8.67(\mathrm{~s}, 1 \mathrm{H}$, $-\mathrm{N}=\mathrm{CH}-), 8.03(\mathrm{~s}, 1 \mathrm{H}$, pyrazole-H), $7.80(\mathrm{~d}, 1 \mathrm{H}, \mathrm{J}=8.00 \mathrm{~Hz}, \mathrm{Ar}-\mathrm{H}), 7.63-7.47(\mathrm{~m}, 6 \mathrm{H}, \mathrm{Ar}-\mathrm{H}), 3.66$ $\left(\mathrm{s}, 3 \mathrm{H}\right.$, pyrazole- $\left.\mathrm{CH}_{3}\right), 2.85\left(\mathrm{~s}, 3 \mathrm{H},-\mathrm{CH}_{3}\right) .{ }^{13} \mathrm{C}-\mathrm{NMR}\left(125 \mathrm{MHz}, \mathrm{DMSO}-d_{6}, \mathrm{ppm}\right) \delta: 171.53,160.76$, 158.52, 141.59, 138.08, 137.68, 135.37, 135.20, 134.23, 132.76, 132.19, 132.08, 131.22, 130.17, 129.94, 128.61, $128.53,127.98,113.75,109.32,108.41,36.62,13.00 . \mathrm{IR}\left(\mathrm{KBr}, \mathrm{cm}^{-1}\right) v: 3444.87,3284.77,3186.40,2929.87$, 1699.29, 1683.86, 1674.21, 1653.00, 1635.64, 1581.63, 1570.06, 1543.05, 1521.84, 1506.41, 1489.05, 1473.62, 1458.18, 1436.97, 1419.61, 1398.39, 1386.82, 1363.67, 1319.31, 1300.02, 1251.80, 1222.87. Anal. Calc. for $\mathrm{C}_{23} \mathrm{H}_{17} \mathrm{Cl}_{3} \mathrm{~N}_{6} \mathrm{O}_{3}$ : C, 51.95\%; H, 3.22\%; N, 15.80\%; Found: C, 51.99\%; H, 3.48\%; N, $15.93 \%$.

Data for 3-(2-chlorophenyl)-5-methyl-N-(1-methyl-4-(2-(4-nitrobenzylidene)hydrazinecarbonyl)-1H-pyrazol5-yl)isoxazole-4-carboxamide (6i). White solid; m.p. $155-156{ }^{\circ} \mathrm{C}$; yield $89.7 \% ;{ }^{1} \mathrm{H}-\mathrm{NMR}(500 \mathrm{MHz}$, DMSO- $\left.d_{6}, \mathrm{ppm}\right) \delta: 11.79(\mathrm{~s}, 1 \mathrm{H}$, isoxazole-CONH-), $10.37(\mathrm{~s}, 1 \mathrm{H}$, pyrazole-CONH-), $8.42(\mathrm{~s}, 1 \mathrm{H}$, $-\mathrm{N}=\mathrm{CH}-), 8.31(\mathrm{~s}, 1 \mathrm{H}$, pyrazole-H), $8.29(\mathrm{~s}, 1 \mathrm{H}, \mathrm{Ar}-\mathrm{H}), 8.14-7.92(\mathrm{~m}, 2 \mathrm{H}, \mathrm{Ar}-\mathrm{H}), 7.61-7.46(\mathrm{~m}, 5 \mathrm{H}$, $\mathrm{Ar}-\mathrm{H}), 3.67\left(\mathrm{~s}, 3 \mathrm{H}\right.$, pyrazole- $\left.\mathrm{CH}_{3}\right), 2.86\left(\mathrm{~s}, 3 \mathrm{H},-\mathrm{CH}_{3}\right) .{ }^{13} \mathrm{C}-\mathrm{NMR}\left(125 \mathrm{MHz}, \mathrm{DMSO}-d_{6}, \mathrm{ppm}\right) \delta: 171.53$, $160.62,158.46,148.23,144.36,141.23,137.77,132.81,132.17,132.09,130.15,130.13,129.40,128.37,128.24$, 127.96, 127.83, 124.65, 124.56, 113.70, 109.35, 36.66, 12.97. IR $\left(\mathrm{KBr}, \mathrm{cm}^{-1}\right)$ v: 3444.87, 3246.20, 3064.89, 2929.87, 1699.29, 1683.86, 1674.21, 1653.00, 1635.64, 1627.92, 1587.42, 1570.06, 1558.48, 1541.12, 1521.84, $1508.33,1489.05,1473.62,1458.18,1436.97,1411.89,1398.39,1375.25,1344.38,1317.38,1253.73,1215.15$. Anal. Calc. for $\mathrm{C}_{23} \mathrm{H}_{18} \mathrm{ClN}_{7} \mathrm{O}_{5}$ : C, $54.39 \%$; $\mathrm{H}, 3.57 \%$; N, $19.30 \%$; Found: $\mathrm{C}, 54.52 \%$; $\mathrm{H}, 3.74 \%$; N, $19.36 \%$.

Data for 3-(2-chlorophenyl)-N-(4-(2-(furan-2-ylmethylene)hydrazinecarbonyl)-1-methyl-1H-pyrazol-5-yl)-5 -methylisoxazole-4-carboxamide (6j). White solid; m.p. $147-149{ }^{\circ} \mathrm{C}$; yield $86.4 \% ;{ }^{1} \mathrm{H}-\mathrm{NMR}(500 \mathrm{MHz}$, DMSO- $\left.d_{6}, \mathrm{ppm}\right) \delta: 11.45(\mathrm{~s}, 1 \mathrm{H}$, isoxazole-CONH-), $10.36(\mathrm{~s}, 1 \mathrm{H}$, pyrazole-CONH-), $8.21(\mathrm{~s}, 1 \mathrm{H}$, $-\mathrm{N}=\mathrm{CH}-), 7.85(\mathrm{~s}, 1 \mathrm{H}$, pyrazole-H) , 7.61-7.47 (m, 5H, Ar-H, furan-H), 6. $91(\mathrm{t}, 1 \mathrm{H}, J=13.15 \mathrm{~Hz}$, furan-H), $6.63\left(\mathrm{~s}, 1 \mathrm{H}\right.$, furan-H), $3.65\left(\mathrm{~s}, 3 \mathrm{H}\right.$, pyrazole- $\left.\mathrm{CH}_{3}\right), 2.85\left(\mathrm{~s}, 3 \mathrm{H},-\mathrm{CH}_{3}\right) .{ }^{13} \mathrm{C}-\mathrm{NMR}(125 \mathrm{MHz}$, DMSO- $d_{6}$, ppm) $\delta: 171.48,163.12,160.69,158.35,149.97,145.59,137.85,137.60,136.76,133.51,132.81$, 132.17, 130.15, 127.96, 127.84, 113.86, 113.72, 112.70, 109.55, 36.63, 12.96. IR (KBr, cm $\left.{ }^{-1}\right)$ v: 3462.22, $3265.49,2929.87,1699.29,1683.86,1647.12,1653.00,1635.64,1627.92,1575.84,1570.06,1558.48,1541.12$, 1521.84, 1496.76, 1489.05, 1473.62, 1436.97, 1419.61, 1398.39, 1361.74, 1319.31, 1300.02, 1253.73, 1213.23. Anal. Calc. for $\mathrm{C}_{21} \mathrm{H}_{17} \mathrm{ClN}_{6} \mathrm{O}_{4}$ : C, $55.70 \%$; H, 3.78\%; N, $18.56 \%$; Found: $\mathrm{C}, 55.78 \%$; H, $4.03 \%$; N, $18.89 \%$.

Data for 3-(2-chlorophenyl)-5-methyl-N-(1-methyl-4-(2-(3,4,5-trimethoxybenzylidene)hydrazine carbonyl)-1H -pyrazol-5-yl)isoxazole-4-carboxamide (6k). White solid; m.p. 206-207 ${ }^{\circ} \mathrm{C}$; yield $91.2 \%$; ${ }^{1} \mathrm{H}-\mathrm{NMR}(500 \mathrm{MHz}$, 
DMSO- $\left.d_{6}, \mathrm{ppm}\right) \delta: 11.44$ (s, 1H, isoxazole-CONH-), 10.28 (s, 1H, pyrazole-CONH-), 8.26 (s, $1 \mathrm{H}$, $-\mathrm{N}=\mathrm{CH}-), 7.80(\mathrm{~s}, 1 \mathrm{H}$, pyrazole-H), 7.60-7.47 (m, 4H, Ar-H), $6.99(\mathrm{~s}, 1 \mathrm{H}, \mathrm{Ar}-\mathrm{H}), 6.97$ (s, 1H, Ar-H), $3.83\left(\mathrm{~s}, 6 \mathrm{H},-\mathrm{OCH}_{3}\right), 3.70\left(\mathrm{~s}, 3 \mathrm{H}\right.$, pyrazole- $\left.\mathrm{CH}_{3}\right), 3.66\left(\mathrm{~s}, 3 \mathrm{H},-\mathrm{OCH}_{3}\right), 2.85\left(\mathrm{~s}, 3 \mathrm{H},-\mathrm{CH}_{3}\right) .{ }^{13} \mathrm{C}-\mathrm{NMR}$ (125 MHz, DMSO-d $d_{6}$, ppm) $\delta: 171.58,161.01,160.59,158.45,158.23,153.73,153.71,147.08,146.67,137.70$, $136.96,132.82,132.17,130.42,130.15,127.95,127.81,114.14,110.70,109.58,104.72,104.44,60.65,56.48$, 36.68, 13.02. IR (KBr, $\left.\mathrm{cm}^{-1}\right) v: 3444.87,3226.91,31867.40,3066.82,2929.87,1699.29,1683.86,1668.43$, 1647.21, 1616.35, 1577.77, 1558.48, 1541.12, 1527.62, 1506.41, 1489.05, 1473.62, 1448.54, 1417.68, 1381.03, $1354.03,1317.38,1301.95,1238.30,1203.58$. Anal. Calc. for $\mathrm{C}_{26} \mathrm{H}_{25} \mathrm{ClN}_{6} \mathrm{O}_{6}: \mathrm{C}, 56.47 \% ; \mathrm{H}, 4.56 \%$; , $15.20 \%$; Found: C, 56.86\%; H, 4.84\%; N, 15.47\%.

Data for 3-(2-chlorophenyl)-5-methyl-N-(1-methyl-4-(2-(propan-2-ylidene)hydrazinecarbonyl)-1H-pyrazol-5-yl) isoxazole-4-carboxamide (61). White solid; m.p. $189-191{ }^{\circ} \mathrm{C}$; yield 87.2\%; ${ }^{1} \mathrm{H}-\mathrm{NMR}$ (500 MHz, DMSO-d ppm) $\delta: 10.16$ (s, 1H, isoxazole-CONH-), 9.28 (s, 1H, pyrazole-CONH-), 7.86 (s, 1H, pyrazole-H), 7.60-7.47 (m, 4H, Ar-H), $4.34\left(\mathrm{~s}, 3 \mathrm{H},-\mathrm{CH}_{3}\right), 3.61\left(\mathrm{~s}, 3 \mathrm{H}\right.$, pyrazole- $\left.\mathrm{CH}_{3}\right), 2.85\left(\mathrm{~s}, 3 \mathrm{H}\right.$, isoxazole- $\left.\mathrm{CH}_{3}\right)$, $2.50\left(\mathrm{~s}, 3 \mathrm{H},-\mathrm{CH}_{3}\right) .{ }^{13} \mathrm{C}-\mathrm{NMR}\left(125 \mathrm{MHz}, \mathrm{DMSO}-d_{6}\right.$, ppm) $\delta: 170.80,161.37,159.91,159.61,136.57,135.96$, $132.09,131.43,131.38,129.43,129.38,127.22,127.15,112.97,108.85,35.82,24.84,18.36,12.22$. IR (KBr, $\mathrm{cm}^{-1}$ ) $v: 3444.87,3066.82,1699.29,1683.86,1668.43,1653.00,1635.64,1616.35,1577.77,1558.48,1541.12$, 1521.84, 1506.41, 1489.05, 1458.18, 1473.62, 1429.25, 1411.89, 1386.82, 1338.60, 1313.52, 1279.88, 1236.73. Anal. Calc. for $\mathrm{C}_{19} \mathrm{H}_{19} \mathrm{ClN}_{6} \mathrm{O}_{3}$ : C, 55.01\%; H, 4.62\%; N, 20.26\%; Found: C, 55.35\%; H, 4.98\%; N, $20.52 \%$.

Data for 3-(2-chlorophenyl)-5-methyl-N-(1-methyl-4-(2-(2-methylpropylidene)hydrazinecarbonyl)-1H-pyrazol -5-yl)isoxazole-4-carboxamide (6m). White solid; m.p. $212-214{ }^{\circ} \mathrm{C}$; yield $88.3 \% ;{ }^{1} \mathrm{H}-\mathrm{NMR}(500 \mathrm{MHz}$, DMSO- $d_{6}$, ppm) $\delta: 11.14(\mathrm{~s}, 1 \mathrm{H}$, isoxazole-CONH-), $9.27(\mathrm{~s}, 1 \mathrm{H}$, pyrazole-CONH-) 7.85 (s, $1 \mathrm{H}$, pyrazole-H), 7.60-7.47 (m, 5H, -C=NH-, Ar-H), $4.32\left(\mathrm{~s}, 3 \mathrm{H}, \mathrm{CH}_{3}\right), 3.61\left(\mathrm{~s}, 3 \mathrm{H}\right.$, pyrazole- $\left.\mathrm{CH}_{3}\right), 2.85$ $\left(\mathrm{s}, 4 \mathrm{H},-\mathrm{CH}-,-\mathrm{CH}_{3}\right), 2.50\left(\mathrm{~s}, 3 \mathrm{H},-\mathrm{CH}_{3}\right) .{ }^{13} \mathrm{C}-\mathrm{NMR}\left(125 \mathrm{MHz}, \mathrm{DMSO}-d_{6}, \mathrm{ppm}\right) \delta: 171.15,166.20$, $161.71,160.25,136.94,136.32,132.43,131.76,131.67,129.74,127.56,127.47,125.47,119.61,113.32,109.20$, $36.14,30.18,20.12,12.57 . \mathrm{IR}\left(\mathrm{KBr}, \mathrm{cm}^{-1}\right)$ v: 3325.28, 3304.06, 3066.82, 1699.29, 1683.86, 1674.21, 1653.00, 1635.64, 1610.56, 1577.77, 1558.48, 1541.12, 1521.84, 1506.41, 1489.05, 1458.18, 1473.62, 1458.18, 1429.25, $1411.89,1386.82,1338.60,1278.81,1249.87,1201.65$. Anal. Calc. for $\mathrm{C}_{20} \mathrm{H}_{21} \mathrm{ClN}_{6} \mathrm{O}_{3}: \mathrm{C}, 56.01 \% ; \mathrm{H}, 4.94 \%$; N, 19.60\%; Found: C, 56.19\%; H, 5.13\%; N, 20.01\%.

Data for 3-(2-chlorophenyl)-5-methyl-N-(1-methyl-4-(2-propylidenehydrazinecarbonyl)-1H-pyrazol-5-yl) isoxazole-4-carboxamide (6n). White solid; m.p. 199-201 ${ }^{\circ} \mathrm{C}$; yield 89.6\%; ${ }^{1} \mathrm{H}-\mathrm{NMR}\left(500 \mathrm{MHz}, \mathrm{DMSO}-d_{6}\right.$, ppm) $\delta: 11.01$ (s, 1H, isoxazole-CONH-), 10.23 (s, 1H, pyrazole-CONH-), 7.91 (s, 1H, pyrazole-H), 7.60-7.39 (m, 5H, -C=NH-, Ar-H), $3.62\left(\mathrm{~s}, 3 \mathrm{H}\right.$, pyrazole- $\left.\mathrm{CH}_{3}\right), 2.82(\mathrm{~s}, 3 \mathrm{H}$, isoxazole-CH$), 2.25(\mathrm{q}$, $\left.2 \mathrm{H}, J=14.90 \mathrm{~Hz},-\mathrm{CH}_{2}-\right), 1.05\left(\mathrm{t}, 3 \mathrm{H}, J=14.90 \mathrm{~Hz},-\mathrm{CH}_{3}\right) .{ }^{13} \mathrm{C}-\mathrm{NMR}\left(125 \mathrm{MHz}, \mathrm{DMSO}-d_{6}, \mathrm{ppm}\right) \delta$ : $172.81,166.66,161.98,153.62,146.78,138.90,137.94,134.20,133.51,131.48,129.28,126.80,121.48,116.59$, $38.00,27.25,14.25,12.62$. IR $\left(\mathrm{KBr}, \mathrm{cm}^{-1}\right) v: 3325.28,3304.06,3066.82,1699.29,1683.86,1674.21,1653.00$, 1635.64, 1615.63, 1577.77, 1558.48, 1541.12, 1521.84, 1506.41, 1489.05, 1473.62, 1456.26, 1436.97, 1417.68, $1398.39,1338.60,1309.31,1269.81,1238.74,1207.29$. Anal. Calc. for $\mathrm{C}_{19} \mathrm{H}_{19} \mathrm{ClN}_{6} \mathrm{O}_{3}$ : C, 55.01\%; $\mathrm{H}, 4.62 \%$; N, 20.26\%; Found: C, 55.36\%; H, 4.87\%; N, 20.54\%.

Data for 3-(2,6-dichlorophenyl)-N-(4-(2-(4-chlorobenzylidene)hydrazinecarbonyl)-1-methyl-1H-pyrazol-5-yl) -5-methylisoxazole-4-carboxamide (6o). White solid; m.p. $168-170{ }^{\circ} \mathrm{C}$; yield $91.3 \%$; ${ }^{1} \mathrm{H}-\mathrm{NMR}(500 \mathrm{MHz}$, DMSO- $\left.d_{6}, \mathrm{ppm}\right) \delta: 11.56(\mathrm{~s}, 1 \mathrm{H}$, isoxazole-CONH), $10.26(\mathrm{~s}, 1 \mathrm{H}$, pyrazole-CONH), $8.32(\mathrm{~s}, 1 \mathrm{H}$, $-\mathrm{CH}=\mathrm{N}-)$, 8.03-8.01 (d, 1H, $J=9.70 \mathrm{~Hz}$, pyrazole-H), 7.74-7.51 (m, 7H, Ar-H), 3.61 (s, 3H, pyrazole- $\left.\mathrm{CH}_{3}\right), 2.92\left(\mathrm{~s}, 3 \mathrm{H}\right.$, isoxazole- $\left.\mathrm{CH}_{3}\right) .{ }^{13} \mathrm{C}$ NMR (125 MHz, DMSO-d 6 , ppm) $\delta: 171.84,163.47$, $159.92,158.61,158.40,145.62,142.82,140.37,137.72,134.95,134.91,133.86,132.91,129.47,129.14,128.96$, $128.93,127.28,127.12,113.60,109.62,36.42,13.17$. IR $\left(\mathrm{KBr}_{\mathrm{cm}}^{-1}\right)$ v: 3444.87, 3176.76, 2951.09, 1699.29, 1683.86, 1653.00, 1635.64, 1616.35, 1605.89, 1575.84, 1558.48, 1541.12, 1521.84, 1506.41, 1489.08, 1473.62, $1448.54,1411.89,1398.39,1319.31,1301.95,1215.15$. Anal. Calc. for $\mathrm{C}_{23} \mathrm{H}_{17} \mathrm{Cl}_{3} \mathrm{~N}_{6} \mathrm{O}_{3}$ : C, 51.95\% $\mathrm{H}$, $3.22 \%$; N, 15.80\%; Found: C, 51.98\%; H, 3.59; N, 15.96\%. 
Data for 3-(2,6-dichlorophenyl)-N-(4-(2-(4-fluorobenzylidene)hydrazinecarbonyl)-1-methyl-1H-pyrazol-5-yl) -5-methylisoxazole-4-carboxamide (6p). White solid; m.p. $154-156{ }^{\circ} \mathrm{C}$; yield $90.6 \%$; ${ }^{1} \mathrm{H}-\mathrm{NMR}(500 \mathrm{MHz}$, DMSO- $d_{6}$, ppm) $\delta: 11.51(\mathrm{~s}, 1 \mathrm{H}$, isoxazole-CONH-), 10.25 (s, 1H, pyrazol-CONH-), 8.33 (s, $1 \mathrm{H}$, $-\mathrm{CH}=\mathrm{N}-), 8.05(\mathrm{~d}, 1 \mathrm{H}, J=18.35 \mathrm{~Hz}$, pyrazole-H), 7.78-7.57 (m, 5H, Ar-H), $7.31(\mathrm{t}, 2 \mathrm{H}, J=17.75 \mathrm{~Hz}$, $\mathrm{Ar}-\mathrm{H}), 3.62\left(\mathrm{~s}, 3 \mathrm{H}\right.$, pyrazole- $\left.\mathrm{CH}_{3}\right), 2.92\left(\mathrm{~s}, 3 \mathrm{H}\right.$, isoxazole- $\left.\mathrm{CH}_{3}\right) .{ }^{13} \mathrm{C}-\mathrm{NMR}\left(125 \mathrm{MHz}, \mathrm{DMSO}-d_{6}, \mathrm{ppm}\right)$ $\delta: 171.60,171.21,163.90,162.81,161.93,159.29,157.87$ (d, 1C, 4-F-Ph-C), 145.21, 142.34, 139.74, 138.51, 137.08, 134.29, 132.26, 130.87, 129.00, 128.30, 126.66, 115.88, 115.71, 112.96, 109.02, 107.44, 35.81, 12.54 . IR $\left(\mathrm{KBr}, \mathrm{cm}^{-1}\right) v: 3446.79,3068.75,2951.09,1699.29,1683.86,1653.00,1635.64,1627.92,1602.85,1575.84$, 1558.48, 1541.12, 1521.84, 1506.41, 1489.08, 1473.62, 1448.54, 1419.61, 1398.39, 1319.31, 1298.09, 1234.44, 1213.23. Anal. Calc. for $\mathrm{C}_{23} \mathrm{H}_{17} \mathrm{Cl}_{2} \mathrm{FN}_{6} \mathrm{O}_{3}$ : C, 53.61\%; H, 3.33\%; N, 16.31\%; Found: C, 53.69\%; H, 3.75\%; $\mathrm{N}, 16.51 \%$.

Data for 3-(2,6-dichlorophenyl)-N-(4-(2-(furan-2-ylmethylene)hydrazinecarbonyl)-1-methyl-1H-pyrazol-5-yl) -5-methylisoxazole-4-carboxamide (6q). White solid; m.p. $255-257{ }^{\circ} \mathrm{C}$; yield $86.5 \%$; ${ }^{1} \mathrm{H}-\mathrm{NMR}(500 \mathrm{MHz}$, DMSO- $d_{6}$, ppm) $\delta: 11.46$ (s, 1H, isoxazole-CONH-), 10.29 (s, 1H, pyrazole-CONH-), 8.22 (s, 1H, $-\mathrm{CH}=\mathrm{N}-), 7.99$ (d, 1H, pyrazole-H), 7.85 (s, 1H, Ar-H), 7.64-7.52 (m, 3H, Ar-H, pyrazole-H, furan-H), $6.91\left(\mathrm{~d}, 1 \mathrm{H}, J=9.75 \mathrm{~Hz}\right.$, furan-H), $6.63\left(\mathrm{~s}, 1 \mathrm{H}\right.$, furan-H), 3.62 (s, 3H, pyrazole- $\left.\mathrm{CH}_{3}\right), 2.93$ (s, 3H, isoxazole- $\left.\mathrm{CH}_{3}\right) .{ }^{13} \mathrm{C}-\mathrm{NMR}\left(125 \mathrm{MHz}, \mathrm{DMSO}-d_{6}, \mathrm{ppm}\right) \delta: 171.82,163.26,159.95,158.32,149.98,145.59$, $140.80,139.31,137.64,136.81,134.92,133.62,132.90,128.94,127.28,113.83,112.70,109.67,107.80,36.44$, 13.16. IR $\left(\mathrm{KBr}, \mathrm{cm}^{-1}\right) v: 3446.79,3068.75,2953.02,1689.64,1653.00,1635.64,1602.85,1558.48,1541.12$, 1521.84, 1506.41, 1489.08, 1473.62, 1431.18, 1409.96, 1398.39, 1330.88, 1317.38, 1255.66, 1220.94. Anal. Calc. for $\mathrm{C}_{21} \mathrm{H}_{16} \mathrm{Cl}_{2} \mathrm{~N}_{6} \mathrm{O}_{4}$ : C, 51.76\%; H, 3.31\%; N, 17.25\%; Found: C, 51.84\%; H, 3.36\%; N, $17.28 \%$.

Data for 3-(2,6-dichlorophenyl)-5-methyl-N-(1-methyl-4-(2-(thiophen-2-ylmethylene)hydrazinecarbonyl) -1H -pyrazol-5-yl)isoxazole-4-carboxamide (6r). White solid; m.p. $248-251{ }^{\circ} \mathrm{C}$; yield $85.9 \%$; ${ }^{1} \mathrm{H}-\mathrm{NMR}(500 \mathrm{MHz}$, DMSO- $d_{6}$, ppm) $\delta: 11.43$ (s, 1H, isoxazole-CONH-), $10.22(\mathrm{~s}, 1 \mathrm{H}$, pyrazole-CONH-), $8.54(\mathrm{~s}, 1 \mathrm{H}$, $-\mathrm{CH}=\mathrm{N}-), 7.98(\mathrm{~s}, 1 \mathrm{H}$, pyrazole-H), 7.65-7.49 (m, 5H, Ar-H, thiophen-H), 7.13 (s, 1H, thiophen-H), 3.61(s, 3H, pyrazole- $\left.\mathrm{CH}_{3}\right), 2.91\left(\mathrm{~s}, 3 \mathrm{H}\right.$, isoxazole- $\left.\mathrm{CH}_{3}\right) .{ }^{13} \mathrm{C}-\mathrm{NMR}\left(125 \mathrm{MHz}, \mathrm{DMSO}-d_{6}, \mathrm{ppm}\right) \delta: 172.35$, $171.82,163.26,159.95,158.64,158.45,158.32,149.98,149.87,145.59,140.80,139.31,137.69,137.64,136.81$, 134.92, 133.62, 132.90, 128.94, 128.64, 127.28, 113.83, 113.61, 113.36, 112.70, 109.67, 107.80, 36.72, 13.16 . IR $\left(\mathrm{KBr}, \mathrm{cm}^{-1}\right) v: 3446.79,3265.49,2953.02,1699.29,1683.86,1653.00,1635.64,1595.13,1575.84,1558.48$, 1541.12, 1521.84, 1506.41, 1489.08, 1473.62, 1458.18, 1436.97, 1398.39, 1373.32, 1315.45, 1294.24, 1251.80, 1222.87. Anal. Calc. for $\mathrm{C}_{21} \mathrm{H}_{16} \mathrm{Cl}_{2} \mathrm{~N}_{6} \mathrm{O}_{3} \mathrm{~S}: \mathrm{C}, 50.11 \%$; H, 3.20\%; N, $16.70 \%$; Found: $\mathrm{C}, 50.28 \%$; $\mathrm{H}, 3.43 \%$; N, $16.76 \%$.

\subsection{Biological Evaluations}

Table 1 shows that most of the title compounds exhibited good antiviral activity against TMV in vivo. Especially, among the title compounds evaluated, compounds $\mathbf{6 a}$ and $\mathbf{6 h}$ exhibited good curative activity against TMV, with inhibition rates of $56.2 \%$ and $55.6 \%$ at $500 \mu \mathrm{g} / \mathrm{mL}$, respectively, which were similar to that of Ningnanmycin (54.6\%). Meanwhile, compounds $\mathbf{6 c}, \mathbf{6 d}, \mathbf{6 n}$, and $\mathbf{6 k}$ exhibited significant protection activity against TMV, with inhibition rates of $66.2 \%, 64.5 \%, 64.2 \%$, and $64.7 \%$ at $500 \mu \mathrm{g} / \mathrm{mL}$, respectively, which were equal to that of Ningnanmycin (63.8\%). In addition, compounds $\mathbf{6 c}, \mathbf{6 0}, \mathbf{6 p}, \mathbf{6 q}$, and $\mathbf{6 r}$ revealed excellent inactivation activities against TMV, with inhibition rates of $91.4 \%, 92.3 \%, 91.7 \%, 93.2 \%$, and $92.6 \%$ at $500 \mu \mathrm{g} / \mathrm{mL}$, respectively, which were similar to that of Ningnanmycin $(92.5 \%)$. 
Table 1. Antiviral activity of the title compounds against TMV at $500 \mu \mathrm{g} / \mathrm{mL}$ in vivo.

\begin{tabular}{cccc}
\hline Compounds & Curative Activity $\mathbf{( \% )} \mathbf{a}^{\mathbf{a}}$ & ${\text { Protection Activity } \mathbf{( \% )} \mathbf{a}^{\mathbf{a}}}$ & Inactivation Activity $\mathbf{( \% )}^{\mathbf{a}}$ \\
\hline $\mathbf{3 a}$ & $20.6 \pm 3.3$ & $30.1 \pm 2.3$ & $42.7 \pm 3.2$ \\
$\mathbf{3 b}$ & $21.5 \pm 1.9$ & $28.9 \pm 3.0$ & $39.4 \pm 2.2$ \\
$\mathbf{6 a}$ & $56.2 \pm 1.3$ & $60.3 \pm 2.1$ & $73.3 \pm 1.4$ \\
$\mathbf{6 b}$ & $48.3 \pm 1.5$ & $56.5 \pm 3.2$ & $81.5 \pm 2.5$ \\
$\mathbf{6 c}$ & $51.4 \pm 2.1$ & $66.2 \pm 2.5$ & $91.4 \pm 2.0$ \\
$\mathbf{6 d}$ & $47.1 \pm 1.4$ & $64.5 \pm 1.7$ & $78.2 \pm 2.1$ \\
$\mathbf{6 e}$ & $44.4 \pm 1.7$ & $45.6 \pm 1.2$ & $61.4 \pm 2.0$ \\
$\mathbf{6 f}$ & $47.7 \pm 2.7$ & $59.3 \pm 1.1$ & $71.8 \pm 2.1$ \\
$\mathbf{6 g}$ & $50.9 \pm 3.3$ & $47.8 \pm 3.4$ & $74.1 \pm 1.5$ \\
$\mathbf{6 h}$ & $55.6 \pm 2.0$ & $64.2 \pm 1.9$ & $84.9 \pm 2.6$ \\
$\mathbf{6 i}$ & $43.3 \pm 3.1$ & $55.3 \pm 2.8$ & $73.6 \pm 2.1$ \\
$\mathbf{6 j}$ & $52.8 \pm 2.2$ & $39.4 \pm 2.4$ & $65.9 \pm 2.2$ \\
$\mathbf{6 k}$ & $51.3 \pm 2.6$ & $64.7 \pm 2.9$ & $81.5 \pm 2.3$ \\
$\mathbf{6}$ & $54.7 \pm 2.1$ & $56.9 \pm 1.2$ & $66.7 \pm 3.2$ \\
$\mathbf{6 m}$ & $33.6 \pm 2.4$ & $40.7 \pm 1.8$ & $54.3 \pm 2.1$ \\
$\mathbf{6 n}$ & $27.5 \pm 2.5$ & $35.8 \pm 2.3$ & $46.5 \pm 2.6$ \\
$\mathbf{6 0}$ & $23.3 \pm 1.1$ & $56.2 \pm 2.3$ & $92.3 \pm 1.8$ \\
$\mathbf{6} \mathbf{p}$ & $40.7 \pm 3.2$ & $49.3 \pm 3.1$ & $91.7 \pm 1.5$ \\
$\mathbf{6 q}$ & $21.9 \pm 2.2$ & $36.3 \pm 1.0$ & $93.2 \pm 1.9$ \\
$\mathbf{6 r}$ & $48.9 \pm 2.0$ & $62.6 \pm 1.2$ & $92.6 \pm 2.2$ \\
Ningnanmycin & $54.6 \pm 1.6$ & $63.8 \pm 2.9$ & $92.5 \pm 1.3$ \\
\hline
\end{tabular}

a Average of three replicates.

As shown in Table 2, compounds $\mathbf{6 a}, \mathbf{6 h}$ and $\mathbf{6 k}$ displayed excellent curative activities against TMV, with the $50 \%$ effective concentration $\left(\mathrm{EC}_{50}\right)$ values of $240.8,255.4$ and $267.4 \mu \mathrm{g} / \mathrm{mL}$, which were even better than that of Ningnanmycin $(286.4 \mu \mathrm{g} / \mathrm{mL})$. Meanwhile, as can be seen from Table 2, compounds 6c, 6d, $\mathbf{6 h}$ and $\mathbf{6 j}$ demonstrated excellent protection activities against TMV, with the EC 50 values of $148.4,184.9,189.7$ and $176.5 \mu \mathrm{g} / \mathrm{mL}$, which were superior to that of Ningnanmycin $(198.2 \mu \mathrm{g} / \mathrm{mL})$. Furthermore, compounds $\mathbf{6 q}$ and $\mathbf{6 r}$, as shown in Table 2, exhibited the best inactivation activity, with the $\mathrm{EC}_{50}$ values of 37.3 and $45.1 \mu \mathrm{g} / \mathrm{mL}$, which were even better than that of Ningnanmycin $(46.3 \mu \mathrm{g} / \mathrm{mL})$.

Table 2. The $\mathrm{EC}_{50}$ values of the testing compounds against TMV in vivo.

\begin{tabular}{cccc}
\hline \multirow{2}{*}{ Compounds } & \multicolumn{3}{c}{$\mathbf{E C}_{\mathbf{5 0}}(\boldsymbol{\mu g} / \mathbf{m L})^{\mathbf{a}}$} \\
\cline { 2 - 4 } & Curative Activity & Protection Activity & Inactivation Activity \\
\hline $\mathbf{6 a}$ & $240.8 \pm 2.2$ & $287.4 \pm 2.3$ & $172.3 \pm 2.1$ \\
$\mathbf{6 b}$ & $457.4 \pm 3.4$ & $369.6 \pm 3.2$ & $102.0 \pm 1.9$ \\
$\mathbf{6 c}$ & $342.6 \pm 4.3$ & $148.4 \pm 3.3$ & $62.2 \pm 1.6$ \\
$\mathbf{6 d}$ & $516.9 \pm 2.5$ & $184.9 \pm 2.0$ & $128.3 \pm 2.7$ \\
$\mathbf{6 f}$ & $505.8 \pm 3.3$ & $323.8 \pm 3.5$ & $194.6 \pm 2.8$ \\
$\mathbf{6 g}$ & $362.4 \pm 1.9$ & $/$ & $150.9 \pm 4.0$ \\
$\mathbf{6 h}$ & $255.4 \pm 2.3$ & $189.7 \pm 2.7$ & $83.9 \pm 3.1$ \\
$\mathbf{6 i}$ & $315.2 \pm 3.6$ & $176.5 \pm 2.8$ & $/$ \\
$\mathbf{6 j}$ & $343.7 \pm 4.2$ & $342.5 \pm 3.6$ & $98.0 \pm 2.6$ \\
$\mathbf{6 k}$ & $267.4 \pm 3.1$ & $381.8 \pm 3.6$ & $247.4 \pm 4.5$ \\
$\mathbf{6 0}$ & $/$ & $509.5 \pm 3.2$ & $56.4 \pm 1.7$ \\
$\mathbf{6 p}$ & $632.3 \pm 5.3$ & $/$ & $64.2 \pm 2.3$ \\
$\mathbf{6 q}$ & $/$ & $224.8 \pm 1.9$ & $37.3 \pm 1.9$ \\
$\mathbf{6 r}$ & $455.3 \pm 4.6$ & $198.2 \pm 2.1$ & $45.1 \pm 2.4$ \\
Ningnanmycin & $286.4 \pm 2.2$ & $46.3 \pm 1.9$ \\
\hline
\end{tabular}

${ }^{a}$ Average of three replicates. 
As an extension of this approach, the structure-activity relationships (SAR) were analyzed on the basis of the activity values in Tables 1 and 2 . First, when $\mathrm{R}^{1}$ and $\mathrm{R}^{2}$ substituent groups were 2-Cl and $\mathrm{H}$, respectively, $\mathrm{R}^{3}$ was substituted with 4-Cl-Ph, 2,4-diCl-Ph, and 3,4,5-triMe-Ph groups, the corresponding target compounds $\mathbf{6 a}, \mathbf{6 h}$ and $\mathbf{6 k}$ exhibited excellent curative activity against TMV, which was superior to those of Ningnanmycin and the other target compounds. Second, when $\mathrm{R}^{1}$ and $\mathrm{R}^{2}$ were substituted with 2-Cl and $\mathrm{H}$ groups, respectively, $\mathrm{R}^{3}$ was 2- $\mathrm{Br}-\mathrm{Ph}, 2-\mathrm{Cl}-\mathrm{Ph}, 2,4-\mathrm{diCl}-\mathrm{Ph}$, and Furyl groups, the corresponding target compounds $\mathbf{6 c} \mathbf{c} \mathbf{6} \mathbf{d}, \mathbf{6 h}$ and $\mathbf{6 j}$ exhibited remarkable protection activity against TMV than those of Ningnanmycin and the other target compounds. Third, when $\mathrm{R}^{1}, \mathrm{R}^{2}$, and $\mathrm{R}^{3}$ substituent groups were 2,6-diCl, $\mathrm{H}$, and Furyl, the corresponding compound $\mathbf{6 q}$ revealed the best inactivation activity which was even better than those of Ningnanmycin and the other target compounds. Finally, as shown in Table 1, compound $\mathbf{6}$ showed better anti-TMV activity than those of compound 3. Therefore, we found that, when the presence of the $-\mathrm{Cl}$ at 2-position or 2,6-positions of phenyl at $R^{1}$ substituent group, the presence of small groups $(-H)$ at $R^{2}$ substituent group, the corresponding compounds presented good antiviral activities. We also found that introducing a hydrazine moiety into the target compounds could enhance the anti-TMV activity, which were also observed in previous studies of Li et al. and Yang [20,24].

\section{Experimental}

\subsection{General Methods}

The melting points of the products were determined on a XT-4 binocular microscope (Beijing Tech Instrument Co., Beijing, China) and were not corrected. The IR spectra were recorded on a Bruker VECTOR 22 spectrometer (Bruker Optics Inc. Billerica, MA, USA) in KBr disk. ${ }^{1} \mathrm{H}-\mathrm{NMR}$ and ${ }^{13}$ C-NMR (solvent DMSO- $d_{6}$ ) spectral analyses were performed on a JEOL-ECX 500 NMR spectrometer (JEOL Ltd., Tokyo,Japan) at room temperature using tetramethylsilane (TMS) as an internal standard. Elemental analysis was performed on an Elementar Vario-III CHN analyser (Elementar, Frankfurt, Germany). Microwave experiments were carried out using a CEM Discover Labmate microwave apparatus (Discover ${ }^{\circledR}$ LabMate instrument, CEM Corporate Matthews, NC, USA). Analytical TLC was performed on silica gel $\mathrm{GF}_{254}$ (200-300 mesh). Column chromatographic purification was carried out using silica gel. Commercial reagents were used as received, unless otherwise indicated. All solvents were dried by standard methods in advance and distilled before use.

\subsection{General Procedure for the Preparation of the Intermediate 5}

As shown in Scheme 1, 3-substituted phenyl-5-methylisoxazole-4-carboxylic acid (0.02 mol) was added to distillated $\mathrm{SOCl}_{2}(50 \mathrm{~mL})$ and reacted at $80{ }^{\circ} \mathrm{C}$ for $5 \mathrm{~h}$ to obtain intermediate 2 . Then, intermediate $2(0.02 \mathrm{~mol})$ was added dropwise to a stirred solution of 5-amino-1-methyl-1H-pyrazole-4-carboxylic acid $(0.02 \mathrm{~mol})$ in anhydrous tetrahydrofuran (THF, $100 \mathrm{~mL}$ ) and trimethylamine, the reaction mixture was stirred at room temperature for $2.0 \mathrm{~h}$. The reaction mixture was poured into cold 5.0\% dilute $\mathrm{HCl}$ solution $(200 \mathrm{~mL})$. The solid obtained was filtered, washed several times with water, dried to give the crude product which was further recrystallized and dried to give intermediate 3 . A mixture of intermediate $3(0.02 \mathrm{~mol})$ and acetic anhydride $(0.2 \mathrm{~mol})$ was heated under reflux for $4.0 \mathrm{~h}$. The solvent was removed under reduced pressure. The residue was washed with water. The separated solid was collected by filtration, washed with water, dried, and recrystallized and dried to give intermediate 4 . To a solution of intermediate $4(10 \mathrm{mmol})$ in THF $(50 \mathrm{~mL}), 6 \mathrm{~mL}$ of $80 \%$ hydrazine hydrate was added slowly at room temperature. Then, the mixture was further reacted at room temperature for $2 \mathrm{~h}$. The solvent was removed under reduced pressure, and the residue was washed with water and anhydrous ethanol to give the crude product, then recrystallized by ethanol and dried under vacuum to give the key intermediate 3-(2-chlorophenyl)-N-(4-(hydrazinecarbonyl)-1-methyl-1H-pyrazol-5-yl)-5-methyl isoxazole-4-carboxamide 5. 


\subsection{General Procedure for the Preparation of the Target Compounds $6 a-6 r$}

As shown in Scheme 1, different aldehydes and ketones $(1 \mathrm{mmol})$ were added to a well-stirred solution of $5(1 \mathrm{mmol})$ in ethanol $(8 \mathrm{~mL})$, and then a few drops of glacial acetic acid were added. The resulting mixture was refluxed for $2 \mathrm{~h}$ to afford a solid, and then filtered and recrystallized from a mixture of ethanol and DMF (1:1 in volume) to gain the title compounds $6 \mathbf{a}-\mathbf{6 r}$.

\subsection{In Vivo Antiviral Activity Test}

\subsubsection{Purification of TMV}

Using the Gooding method [37], the virus was multiplied in N. tabacum cv. K326 and purified. The absorbance values were estimated at $260 \mathrm{~nm}$ using an ultraviolet spectrophotometer. The virus concentration was calculated by the following formula, where E represents the extinction coefficient for TMV, $E^{0.1 \%} 1 \mathrm{~cm}{ }^{260 \mathrm{~nm}}$ is 3.1 :

$$
\text { Virus concentration }(\mathrm{mg} / \mathrm{mL})=\left(\mathrm{A}_{260} \times \text { dilution ratio }\right) / E^{0.1 \%}{ }_{1 \mathrm{~cm}}^{260 \mathrm{~nm}}
$$

\subsubsection{Protection Activity of the Title Compounds against TMV in Vivo}

The compound solution was smeared on the left side of growing N. tabacum L. leaves of the same age. The solvent without compound solution was smeared on the right side of the leaves to serve as the control. The leaves, which were previously scattered with silicon carbide, were inoculated with the virus after $12 \mathrm{~h}$ using a brush dipped in $6 \times 10^{-3} \mathrm{mg} / \mathrm{mL}$ TMV and subsequently washed with water and rubbed softly along the nervature once or twice. After three to four days of the inoculation, the local lesions were counted. Three replications were reproduced for each compound.

\subsubsection{Curative Activity of the Title Compounds against TMV in Vivo}

The leaves were inoculated with TMV (concentration of $6 \times 10^{-3} \mathrm{mg} / \mathrm{mL}$ ) by dipping and brushing the whole leaves, which were previously scattered with silicon carbide. The leaves were then washed with water after inoculation for $0.5 \mathrm{~h}$. The compound solution was smeared on the left side of the leaves, and the solvent without compound solution was smeared on the right side for control. The number of local lesions was counted and recorded three to four days after the inoculation. Three replications were reproduced for each compound.

\subsubsection{Inactivation Activity of the Title Compounds against TMV in Vivo}

The virus was inhibited after it was mixed with a compound solution of the same volume for $30 \mathrm{~min}$. The right side of the N. tabacum L. leaves was then inoculated with the solvent and virus mixture for control. All of the leaves were previously scattered with silicon carbide. The number of local lesions was recorded three to four days after the inoculation. Three replications were reproduced for each compound.

The inhibition rates of the compounds were calculated according to the following formula:

Inhibition rate $(\%)=[$ (average local lesion number of control (not treated with compounds) - average local lesion number smeared with compounds)/average local lesion number of control (not treated with compounds) $] \times 100 \%$

\section{Conclusions}

In summary, a series of novel pyrazole-hydrazone derivatives containing an isoxazole moiety were obtained with moderate yields and their antiviral activities against TMV were examined by a half-leaf method. Bioassay results revealed that some of the target compounds exhibited better antiviral activity against TMV in vivo than Ningnanmycin. Among the title compounds, compound 
6a exhibited better curative activity against TMV than that of Ningnanmycin. Meanwhile, compound 6c showed remarkable protection activity against TMV, which was superior to that of Ningnanmycin. Moreover, compound $\mathbf{6 q}$ exhibited better inactivation activity against TMV which were superior to that of Ningnanmycin. The antibacterial tests showed that introducing a hydrazine moiety into the target compounds could enhance the anti-TMV activity; meanwhile, when the presence of the $-\mathrm{Cl}$ at 2-position or 2,6-positions of phenyl in the $\mathrm{R}^{1}$ substituent group, the presence of small groups $(-\mathrm{H})$ in the $\mathrm{R}^{2}$ substituent group, the corresponding compounds presented good antiviral activities. Therefore, this study demonstrated that this series of pyrazole-hydrazone derivatives containing an isoxazole moiety can be considered for further development as a new class of tobacco protection agents.

Supplementary Materials: The ${ }^{1} \mathrm{H}-\mathrm{NMR}$ and ${ }^{13} \mathrm{C}-\mathrm{NMR}$ for all the synthesized compounds are available online. Author Contributions: Writing-Original Draft Preparation, Z.Y.; Writing-Review and Editing, P.L. and X.G.; Project Administration, Z.Y.

Funding: This research was funded by the National Natural Science Foundation of China (Grant No. 21466031).

Conflicts of Interest: The authors declare no conflict of interest.

\section{References}

1. Xie, L.H.; Lin, Q.Y.; Wu, Z.J. Plant Virus; China Press: Beijing, China, 2009; pp. 1-5.

2. Deng, G.B.; Wan, B.; Hu, H.Z.; Chen, J.R.; Yu, M.Q. Biological activity of ningnanmycin on tobacco mosaic virus. Chin. J. Appl. Environ. Biol. 2004, 10, 695-698.

3. Wang, Z.W.; Wang, L.; Ma, S.; Liu, Y.X.; Wang, L.Z.; Wang, Q.M. Design, synthesis, antiviral activity, and SARs of 14-aminophenanthroindolizidines. J. Agric. Food Chem. 2012, 60, 5825-5831. [CrossRef] [PubMed]

4. Song, B.A.; Yang, S.; Jin, L.H.; Bhadury, P.S. Environment-Friendly Antiviral Agents for Plants; Springer: Berlin, Germany, 2010; pp. 1-7.

5. Yu, L.G.; Ni, T.F.; Gao, W.; He, Y.; Wang, Y.Y.; Cui, H.W.; Yang, C.G.; Qiu, W.W. The synthesis and antibacterial activity of pyrazole-fused tricyclic diterpene derivatives. Eur. J. Med. Chem. 2015, 90, 10-20. [CrossRef] [PubMed]

6. Mabkhot, Y.N.; Kaal, N.A.; Alterary, S.; Al-Showiman, S.S.; Barakat, A.; Ghabbour, H.A.; Frey, W. Synthesis, in-vitro antibacterial, antifungal, and molecular modeling of potent anti-microbial agents with a combined pyrazole and thiophene pharmacophore. Molecules 2015, 20, 8712-8729. [CrossRef] [PubMed]

7. Sangani, C.B.; Makwana, J.A.; Duan, Y.T.; Tarpada, U.P.; Patel, Y.S.; Patel, K.B.; Dave, V.N.; Zhu, H.L. Design, synthesis, and antibacterial evaluation of new Schiff's base derivatives bearing nitroimidazole and pyrazole nuclei as potent E. coli FabH inhibitors. Res. Chem. Intermed. 2015, 41, 10137-10149. [CrossRef]

8. Du, S.J.; Tian, Z.M.; Yang, D.Y.; Li, X.Y.; Li, H.; Jia, C.Q.; Che, C.L.; Wang, M.; Qin, Z.H. Synthesis, antifungal activity and structure-activity relationships of novel 3-(difluoromethyl)-1-methyl-1H-pyrazole-4-carboxylic acid amides. Molecules 2015, 20, 8395-8408. [CrossRef] [PubMed]

9. Sun, J.L.; Zhou, Y.M. Synthesis and antifungal activity of the derivatives of novel pyrazole carboxamide and isoxazolol pyrazole carboxylate. Molecules 2015, 20, 4383-4394. [CrossRef] [PubMed]

10. Wang, S.L.; Shi, Y.J.; He, H.B.; Li, Y.; Li, Y.; Dai, H. Synthesis and bioactivity of novel pyrazole oxime derivatives containing oxazole ring. Chin. Chem. Lett. 2015, 26, 672-674. [CrossRef]

11. Dai, H.; Ye, L.Y.; Zhuang, H.Y.; Dai, B.J.; Fang, Y.; Shi, Y.J. Design, synthesis and bioactivities of novel dichloro-allyloxy-phenol-containing pyrazole oxime derivatives. Molecules 2015, 20, 21870-21880. [CrossRef] [PubMed]

12. Lv, X.H.; Xiao, J.J.; Ren, Z.L.; Chu, M.J.; Wang, P.; Meng, X.F.; Li, D.D.; Cao, H.Q. Design, synthesis and insecticidal activities of $N$-(4-cyano-1-phenyl- $1 H$-pyrazol-5-yl)-1,3-diphenyl-1H-pyrazole-4-carboxamide derivatives. RSC Adv. 2015, 5, 55179-55185. [CrossRef]

13. Deng, X.L.; Xie, J.; Li, Y.Q.; Yuan, D.K.; Hu, X.P.; Li, Z.; Wang, Q.M.; Chi, M.; Yang, X.L. Design, synthesis and biological activity of novel substituted pyrazole amide derivatives targeting EcR/USP receptor. Chin. Chem. Lett. 2016, 27, 566-570. [CrossRef] 
14. Singh Jadav, S.; Nayan Sinha, B.; Pastorino, B.; de Lamballerie, X.; Hilgenfeld, R.; Jayaprakash, V. Identification of pyrazole derivative as an antiviral agent against Chikungunya through HTVS. Lett. Drug Des. Discov. 2015, 12, 292-301. [CrossRef]

15. El-Sabbagh, O.I.; Baraka, M.M.; Ibrahim, S.M.; Pannecouque, C.; Andrei, G.; Snoeck, R.; Balzarini, J.; Rashad, A.A. Synthesis and antiviral activity of new pyrazole and thiazole derivatives. Eur. J. Med. Chem. 2009, 44, 3746-3753. [CrossRef] [PubMed]

16. Ma, H.J.; Zhang, J.H.; Xia, X.D.; Kang, J.; Li, J.H. Design, synthesis and herbicidal evaluation of novel 4-(1H-pyrazol-1-yl)pyrimidine derivatives. Pest Manag. Sci. 2015, 71, 1189-1196. [CrossRef] [PubMed]

17. Kang, J.; Yue, X.L.; Chen, C.S.; Li, J.H.; Ma, H.J. Synthesis and herbicidal activity of 5-heterocycloxy-3-methyl-1-substituted-1H-pyrazoles. Molecules 2016, 21, 39. [CrossRef] [PubMed]

18. Kang, S.H.; Song, B.A.; Wu, J.; He, M.; Hu, D.Y.; Jin, L.H.; Zeng, S.; Xue, W.; Yang, S. Design, synthesis and insecticidal activities of novel acetamido derivatives containing $N$-pyridylpyrazole carboxamides. Eur. J. Med. Chem. 2013, 67, 14-18. [CrossRef] [PubMed]

19. Wu, J.; Kang, S.H.; Song, B.A.; Hu, D.Y.; He, M.; Jin, L.H.; Yang, S. Synthesis and antifungal activities of novel nicotinamide derivatives containing 1,3,4-oxadiazole. Chem. Cent. J. 2013, 7, 64. [CrossRef] [PubMed]

20. Li, L.; Li, Z.; Wang, K.L.; Liu, Y.X.; Li, Y.Q.; Wang, Q.M. Synthesis and antiviral, insecticidal, and fungicidal activities of gossypol derivatives containing alkylimine, oxime or hydrazine moiety. Bioorg. Med. Chem. 2016, 24, 474-483. [CrossRef] [PubMed]

21. Yang, X.D. Synthesis and biological activity of hydrazone derivatives containing pyrazole. J. Chem. Res. 2008, 9, 489-491. [CrossRef]

22. Yang, Z.B.; Hu, D.Y.; Zeng, S.; Song, B.A. Novel hydrazone derivatives containing pyridine amide moiety: Design, synthesis, and insecticidal activity. Bioorg. Med. Chem. Lett. 2016, 26, 1161-1164. [CrossRef] [PubMed]

23. Wu, J.; Xie, D.D.; Shan, W.L.; Zhao, Y.H.; Zhang, W.; Song, B.A.; Yang, S.; Ma, J. Synthesis and insecticidal activity of anthranilic diamides with hydrazone substructure. Chem. Pap. 2015, 69, 993-1003. [CrossRef]

24. Liu, Y.X.; Song, H.J.; Huang, Y.Q.; Li, J.R.; Zhao, S.; Song, Y.C.; Yang, P.W.; Xiao, Z.X.; Liu, Y.X.; Li, Y.Q.; et al. Design, synthesis, and antiviral, fungicidal, and insecticidal activities of tetrahydro- $\beta$-carboline-3-carbohydrazide derivatives. J. Agric. Food Chem. 2014, 62, 9987-9999. [CrossRef] [PubMed]

25. Liu, Y.; Lu, B.W.; Xin, J.R.; Li, J.F.; Wu, J.B.; Bao, X.R. Synthesis and antibacterial activities of N-[(1-aryl-3-phenyl-pyrazol-4-yl)methylene]-2-(halo-o-hydroxyphenyl)hydrazide derivatives. Chem. Res. Chin. Univ. 2013, 29, 449-453. [CrossRef]

26. Xing, M.; Zhao, T.T.; Ren, Y.J.; Peng, N.N.; Yang, X.H.; Li, X.; Zhang, H.; Liu, G.Q.; Zhang, L.R.; Zhu, H.L. Synthesis, biological evaluation, and molecular docking studies of pyrazolyl-acylhydrazone derivatives as novel anticancer agents. Med. Chem. Res. 2014, 23, 3274-3286. [CrossRef]

27. Nasrullah, M.; Khan, M.A.; Khan, M.N.; Humphrey, M.G.; Nasim, F.H.; Abidi, M.G.; Khan, M.N.; Farooq, U.; Munawar, M.A. Diaryl pyrazole-4-carbaldehyde benzoylhydrazones metal complexes: Synthesis and their antibacterial and antioxidant screening. Asian J. Chem. 2013, 25, 419-423. [CrossRef]

28. El-Sayed, M.A.; Abdel-Aziz, N.I.; Abdel-Aziz, A.A.; El-Azab, A.S.; Asiri, Y.A.; ElTahir, K.E.H. Design, synthesis, and biological evaluation of substituted hydrazone and pyrazole derivatives as selective COX-2 inhibitors: Molecular docking study. Bioorg. Med. Chem. 2011, 19, 3416-3424. [CrossRef] [PubMed]

29. Abadi, A.H.; Eissa, A.A.H.; Hassan, G.S. Synthesis of novel 1,3,4-trisubstituted pyrazole derivatives and their evaluation as antitumor and antiangiogenic agents. Chem. Pharm. Bull. 2003, 51, 838-844. [CrossRef] [PubMed]

30. Fraga, A.G.M.; Rodrigues, C.R.; Miranda, A.L.P.; Barreiro, E.J.; Fraga, C.A.M. Synthesis and pharmacological evaluation of novel heterotricyclic acylhydrazone derivatives, designed as PAF antagonists. Eur. J. Pharm. Sci. 2000, 11, 285-290. [CrossRef]

31. Kang, Y.K.; Shin, K.J.; Yoo, K.H.; Seo, K.J.; Hong, C.Y.; Lee, C.S.; Park, S.Y.; Kim, D.J.; Park, S.W. Synthesis and antibacterial activity of new carbapenems containing isoxazole moiety. Bioorg. Med. Chem. Lett. 2000, 10, 95-99. [CrossRef]

32. Santos, M.M.M.; Faria, N.; Lley, J.; Coles, S.J.; Hursthouse, M.B.; Martins, M.L.; Moreira, R. Reaction of naphthoquinones with substituted nitromethanes. Facile synthesis and antifungal activity of naphtho[2,3-d]isoxazole-4,9-diones. Bioorg. Med. Chem. Lett. 2010, 20, 193-195. [CrossRef] [PubMed] 
33. Zhang, D.W.; Lin, F.; Li, B.C.; Liu, H.W.; Zhao, T.Q.; Zhang, Y.M.; Gu, Q. Efficient synthesis of bis-isoxazole ethers via 1,3-dipolar cycloaddition catalysed by $\mathrm{Zn} / \mathrm{Zn}^{2+}$ and their antifungal activities. Chem. Pap. 2015, 69, 1500-1511. [CrossRef]

34. Yu, X.L.; Liu, Y.X.; Li, Y.Q.; Wang, Q.M. Design, synthesis, and acaricidal/insecticidal activities of oxazoline derivatives containing a sulfur ether moiety. J. Agric. Food Chem. 2015, 63, 9690-9695. [CrossRef] [PubMed]

35. Liu, G.; Ozoe, F.; Furuta, K.; Ozoe, Y. 4,5-Substituted 3-isoxazolols with insecticidal activity act as competitive antagonists of housefly GABA receptors. J. Agric. Food Chem. 2015, 63, 6304-6312. [CrossRef] [PubMed]

36. Daghigh, L.R.; Pordel, M.; Davoodnia, A.; Jajarmi, M. Synthesis, antiviral, and cytotoxic investigation of imidazo[4,5-a]acridones. Med. Chem. Res. 2015, 24, 3912-3919. [CrossRef]

37. Gooding, G.V., Jr.; Hebert, T.T. A simple technique for purification of tobacco mosaic virus in large quantities. Phytopathology 1967, 57, 1285. [PubMed]

Sample Availability: Samples of the compounds 6a-6r are available from the authors.

(C) 2018 by the authors. Licensee MDPI, Basel, Switzerland. This article is an open access article distributed under the terms and conditions of the Creative Commons Attribution (CC BY) license (http://creativecommons.org/licenses/by/4.0/). 